\title{
The Effects of Eight Weeks Upper Body and Lower Body Resistance Training on Plasma Adiponectin and Leptin Levels in Young Men
}

\author{
${ }^{1}$ Masoud Zamani, ${ }^{1}$ Maghsoud Peeri* \\ ${ }^{1}$ Department of Exercise Physiology, Faculty of Sport Science, Tehtan Central Branch, Islamic Azad University, \\ Tehran, Iran.
}

\begin{abstract}
Background. Adipose tissue secrets various hormones in body such as adiponectin which has close relationship with controlling weight.

Objectives. Therefore, the present article determines movement pattern effect of resistance training on adiponectin, in young men.

Methods. 40 male untrained students in $23.8 \pm 2.66$ years old and weight of $67.43 \pm 4.96 \mathrm{~kg}$ were randomly selected, who participated volunteering in this investigation, from upper body exercise, lower body exercise, combined (three resistance exercise sessions were held in each week during 8 weeks, 5 exercise sets with $60-85 \%$ of one repetition maximum) and control exercise $(\mathrm{n}=10)$. Blood samples were measured completely similarly in three steps of pre-test, fourth week, and one day after the eighth week exercise analyzed.

Results. It is shown that in upper body group after the 8th week, adipose percentage reduced $7.39 \%(\mathrm{P}<0.002)$ and leptin was decreased up to $64.02 \%(\mathrm{P}<0.001)$, adiponectin significantly increased $90.42 \%(\mathrm{P}<0.001)$ than pre-test step. In addition, in lower body group, adipose percentage reduced $7.39 \%(\mathrm{P}<0.006)$ and leptin decreased 56.95\% $(\mathrm{P}<0.001)$, adiponectin significantly increased $87.82 \%(\mathrm{P}<0.012)$, than pre-test step. In combined group, body mass index (BMI) increased 1.88\% ( $\mathrm{P}<0.006)$, muscular mass increased $2.24 \%(\mathrm{P}<0.007)$, and adiponectin significantly increased $91.56 \%(\mathrm{P}<0.001)$, leptin $(\mathrm{P}<0.001)(59.3 \%)$ after eight weeks training significantly decreased than pretest step.

Conclusion. Various resistance exercises, increase adiponectin and leptin that are associated with change body composition. These changes can be effective for prevention of cardiovascular diseases.
\end{abstract}

KEY WORDS: Resistance Training, Adiponectin, Leptin.

\section{INTRODUCTION}

Today, obesity is the main problem in the world and its popularity is increasing both in advanced and the third world countries significantly $(1,2)$. Many efforts have been made to control or prevent this global epidemic (2). On the other hand, adipose tissue secrets important proteins called adipo-cytokines having various biological effects. One of adipo-cytokines is adiponectin that may be an important index for cardiovascular disease (3). It seems that adiponectin has anti-atherosclerosis and antiatherogenic effects (4). While resistance exercises effect on various studies has contradicted results (5). For example, some reported that 12 weeks resistance exercise in average intensity, while others showed that 12

*. Corresponding Author:

Maghsoud Peeri

E-mail: mpeeri@iauctb.ac.ir 
weeks of resistance exercise doesn't change plasma adiponectin significantly (6). On the other hand, Some studies have shown that aerobic exercises reduce or don't change leptin amount (7). While research on resistance exercise effect on leptin concentration are contradicted and limited. For example, Lau et al. found that 6 weeks of resistance exercise in contrary of increasing muscular power doesn't significantly influence on body composition and leptin conception (8) while in Ibez et al., studied progressive resistance exercises reduce plasma leptin in obese women significantly (9), Therefore, in spite of different effects of resistance exercises from other exercises on various factors, many effectiveness aspects of resistance exercises on adipokines haven't identified yet. On the other hand, resistance exercise focus on upper body and lower body parts in different days and sessions. The used protocols by body builders (10) that may be accepted highly adolescents and teenagers can be this exercise training. According to probable role of adipo-cytokines and testosterone as relationship between body activity and cardiovascular disease (5) and regarding to contradictions and limitations of results, the present study aims on determination effects of 8 weeks of upper and lower body parts resistance exercise and combination on adiponectin and leptin, in Young Men.

\section{MATERIALS AND METHODS}

Participants. 40 unexercised healthy men (with average age of $23.8 \pm 2.66$ years old and weight of $67.43 \pm 4.96 \mathrm{~kg}$ ) were volunteered of participation in this study (Table 1). They signed the written consent form. Studied people didn't practice in any sport exercise al least for 2 years. All participators were healthy according to cardiac, respiratory, renal, and metabolic aspect and have no orthopedic damage preventing their body activity and their health should be proved by doctor. Participators were put in all four groups by simple random method.

Study Design. Resistance exercise protocol in the present study includes 8 weeks practice with weight. Three sessions of exercise in week was implemented every other day. Exercise duration with weight was about $65 \mathrm{~min}$ in each session. To reduce some confounding factors affecting results of the study and to reduce the effects of food type on adiponectin and leptin subjects were asked at Three days before testing night, meals in every days were provided to remove effect of nutrition on studied variables (55\% carbohydrates, $15 \%$ protein, and $30 \%$ fat). They were asked to sleep about 11 o'clock at night. Blood samples were collected before starting the protocol, at the end of the fourth week, and the day after the last exercise session of the eight week after 12 hours of fasting. Samples were analyzed according to serum level of leptin, adiponectin. Participators referred to laboratory after 12 hours fasting and $5 \mathrm{cc}$ blood was gotten from participators from right hand antecubital vein in the fourth week and at the end of the eighth week in sitting position. Taking samples from exercise groups were taken in the same day hour to prevent from fasting effects and day-night rhythm.

Training Protocol. Exercise with weight for upper body parts include bench press, rowing, shoulder press, lat pull down, Biceps curl, and triceps curl and exercise for lower body parts include squat, launch, leg press, leg extensions, leg curl, and seated calf raise, and combined exercises include bench press, leg press, lat pull down, squat, biceps curl, seated calf raise that in each station one turn with $60 \%$ of maximum power with 8-12 repetition and 4 set with 6-8 repetition were conducted by $80-85 \%$ of maximum power. Rest time between sets was 2 minutes and between movements was 3 minutes. The intensity of exercise in the fourth week reduced for proper recovery and prevention from overtraining and participators continued their exercises after determining maximum power with $80-85 \%$ of new maximum power. Participators did jogging and stretching exercises about 10 minutes at the beginning of each session for warm-up before starting training with weights. At the end of each session, they again did jogging and stretching exercises to slow down the repetition. Participators of exercise group were asked to prevent any exercise in research duration except the prescribed exercise. Participators of control group avoided any physical exercise extra of their daily life.

Measurements. Starting time of each session exercise was adjusted in a way that taken blood sample was obtained at least at 19 hours 
recovery in the same time for all sessions. Blood samples were centrifuged at 3000 RPM for 10 minutes to separate serum and the obtained serum was distributed and immediately frozen at $-80^{\circ} \mathrm{C}$ for later analysis. Biochemical analysis for measuring serum leptin was performed by ELISA method with 10 picogram $/ \mathrm{mg} / 1$ sensitivity by using MICROPLAT and Kit made by BOSTER Co (USA). and adiponectin with less than 60 pico-gram sensitivity by ELIZA method using MICROPLATE kit of Boster Company (USA). In the first day of this study, participators referred to gym to evaluate and register BMI, body composition, 1 repetition maximum (1RM) for resistance exercises. Body composition of participators was analyzed using Body composition analyzer BC-418MA system by considering usage principles and values were reported as fat percentage and muscular mass. In order to measure 1RM, participators warmed up by light weights (about $50 \%$ of a predicted $\mathrm{RM}$ ) then tried for a weight increase with $85 \%$ of RM. rest (3-5min) was between 2 exercises. The last lifted weight was considered as 1RM.

Table 1. Personal feature of control and exercise group (Mean+SD)

\begin{tabular}{llllll}
\hline Exercise group & Age (year) & Height $(\mathrm{cm})$ & Weight $(\mathrm{kg})$ & Body fat, \% & BMI $\left(\mathrm{kg} / \mathrm{m}^{2}\right)$ \\
\hline Upper body & $24.15 \pm 2.77$ & $170.70 \pm 6.76$ & $70 \pm 3.32$ & $15.6 \pm 4.83$ & $23.00 \pm 1.49$ \\
\hline Lower body & $21.00 \pm 0.79$ & $174.60 \pm 8.22$ & $65.13 \pm 7.39$ & $15.7 \pm 4.85$ & $23.50 \pm 3.15$ \\
\hline Combination & $21.20 \pm 0.76$ & $176.20 \pm 1.55$ & $66.52 \pm 3.96$ & $19.1 \pm 3.30$ & $25.00 \pm 2.83$ \\
\hline Control & $22.26 \pm 2.23$ & $175.20 \pm 5.07$ & $68.10 \pm 3.96$ & $15.7 \pm 4.88$ & $23.90 \pm 2.13$ \\
\hline
\end{tabular}

BMI: Body Mass Index

Statistical Analysis. Data was expressed by Mean \pm SD. To analyze research data after determining participators normality, variance test with repeated measurement and equal to its nonparametric, Friedman test, if data is not normal and one-way variance analysis inside group and Kruskal-Wallis were used. In order to determine intergroup differences, post hoc Bonferroni test, Scheffe, 2K, and Umann-Whitney by modification of Bonferroni and Wilcoxon's post hoc test (as post hoc test of Friedman statistical method with Bonferroni modification were used. Statistical analysis was performed by software SPSS20at significant level $\mathrm{P}<0.05$.

\section{RESULTS}

Results of research showed that in upper body group. Fat percent reduced after 8 weeks $(\mathrm{P}<0.002)$, adiponectin increases $(\mathrm{P}<0.000)$, Leptin were reduced significantly $(\mathrm{P}<0.000)$, than pre-test step. In addition, in lower body group, fat percent reduced $(\mathrm{P}<0.006)$, adiponectin increased $(\mathrm{P}<0.012)$, leptin reduced significantly $(\mathrm{P}<0.000)$, than pre-test step. In combined group, BMI $(\mathrm{P}<0.006)$, Muscle mass $(\mathrm{P}<0.007), \quad$ and adiponectin $\quad(\mathrm{P}<0.000)$ significantly increased, leptin reduced after 8 weeks $(\mathrm{P}<0.000)$ than pre-test step. In the rest, amount of small adiponectin change is different in research groups $(\mathrm{P}<0.000)$ and upper body, lower body, and combined exercises increased significantly than control group $(\mathrm{P}<0.008)$. Leptin change amount was different in research groups after 8 weeks $(\mathrm{P}<0.000)$ and upper body, lower body, and combined exercises significantly reduced leptin than control group $(\mathrm{P}<0.05)$. Changes in fat percent were different in research groups after 8 weeks $(\mathrm{P}<0.03)$ and this difference was between fat percent in combined and upper body exercises $(\mathrm{P}<0.008)$. Changes in BMI was different in research groups after 8 weeks $(\mathrm{P}<0.007)$ and this difference was just between combined and upper body exercises $(\mathrm{P}<0.008)$ (Table 2).

Increases of 1RM in the upper body group were observed in the chest press and arm curl $(\mathrm{P}<0.000)$. Increases for each of the exercises were $20 \%$ in chest press and $30 \%$ in arm curl, On the other hand Increases of 1RM in the lower body group were observed in the squat, seated calf raise $(\mathrm{P}<0.00)$ Increases for each of the exercises were $15 \%$ in squat, $20 \%$ in seated calf raise. $1 \mathrm{RM}$ in the in combined group were observed in the chest press and arm curl $(\mathrm{P}<0.00)$. Increases for each of the exercises were $15 \%$ in chest press and $25 \%$ in arm curl,and increases of $1 \mathrm{RM}$ in the lower body group were 
observed in the squat, seated calf raise $(\mathrm{P}<0.00)$

Increases for each of the exercises were $12 \%$ in squat, $20 \%$ in seated calf raise.

Table 2. anthropometric (physiological) measures

\begin{tabular}{|c|c|c|c|c|c|c|}
\hline \multicolumn{2}{|l|}{ Variables } & \multirow{3}{*}{$\begin{array}{l}\frac{\text { Group }}{\text { Pretest }} \\
\text { Mean } \pm \text { SD }\end{array}$} & \multicolumn{2}{|c|}{ Weeks of Training } & \multicolumn{2}{|r|}{$\mathbf{p}$} \\
\hline & & & Fourth week & Eighth Week & \multirow{2}{*}{ Within Group } & \multirow{2}{*}{ Between Group } \\
\hline & & & Mean \pm SD & Mean \pm SD & & \\
\hline \multirow{4}{*}{ BMI } & Upper E. & $2.76 \pm 24.15$ & $23.78 \pm 3.09$ & $23.91 \pm 2.73$ & \multirow{4}{*}{$0.006^{*}$} & \multirow{4}{*}{$0.007^{* *}$} \\
\hline & Lower E. & $21.10 \pm 0.79$ & $21.10 \pm 0.54$ & $21.16 \pm 0.49$ & & \\
\hline & Combined & $21.38 \pm 0.76$ & $21.72 \pm 0.66$ & $\dagger 21.79 \pm 0.61$ & & \\
\hline & Control & $22.26 \pm 2.23$ & $21.18 \pm 0.92$ & $21.17 \pm 1.04$ & & \\
\hline \multirow{4}{*}{ Body fat \% } & Upper E. & $18.58 \pm 2.25$ & $17.87 \pm 1.78$ & $\dagger 17.30 \pm 1.86$ & $0.002^{*}$ & \multirow{4}{*}{$0.030^{* *}$} \\
\hline & Lower E. & $13.53 \pm 0.84$ & $13.61 \pm 0.65$ & $\dagger 12.63 \pm 0.79$ & $0.006^{*}$ & \\
\hline & Combined & $12.78 \pm 2.41$ & $12.63 \pm 2.02$ & $12.83 \pm 1.59$ & & \\
\hline & Control & $17.26 \pm 5.54$ & $17.16 \pm 5.23$ & $17.24 \pm 5.07$ & & \\
\hline \multirow{4}{*}{ Muscle Mass } & Upper E. & $53.73 \pm 1.24$ & $53.93 \pm 1.86$ & $54.64 \pm 1.62$ & \multirow{4}{*}{$0.007^{*}$} & \multirow{4}{*}{0.173} \\
\hline & Lower E. & $49.79 \pm 6.31$ & $49.94 \pm 5.98$ & $50.51 \pm 5.88$ & & \\
\hline & Combined & $54.91 \pm 1.33$ & $56.11 \pm 1.72$ & $\dagger 56.17 \pm 1.29$ & & \\
\hline & Control & $50.55 \pm 4.46$ & $50.18 \pm 5.81$ & $50.17 \pm 6.11$ & & \\
\hline
\end{tabular}

*: significant difference three test steps $(\mathrm{p}<0.05), \uparrow:$ significant difference from pre-test (for BMI and muscular mass) and significant difference from amounts of 4 weeks for fat $\%(p<0.025)$, **: significant difference among four groups $(\mathrm{p}<0.05)$.

\section{DISCUSSION}

Results of some studies in proving results of the present research reported significant increase of adiponectin concentration after resistance exercises in medium and intensive levels (5) In a study, effect of 12 various exercise months were examined on inflammatory indexes in diabetes type II and metabolic syndrome that adiponectin level increased in exercise group (with intensive aerobic exercises and aerobic exercises +resistance exercises) (11). Yet, results of the resent study disagreed with results of Ahmadizad et al. study who reported that 12 weeks of power exercise (including 11 movement stations circularly and 3 days in week for 12 weeks, each session about 60 minutes and intensity of 50$60 \%$ of $1-\mathrm{RM}$ of participators) and resistance exercise (running in $75-85 \%$ of maximum heart rate, 3 days in week, for 12 weeks) don't significantly change plasma adiponectin in participators (6). They claimed that maybe low intensity of exercises is for non-change of adiponectin in people. One of probable reasons of disagreement off these results can be found in age, gender, weight, and different amounts of BMI, and exercise protocol differences $(9,12)$ As it was observed, resistance exercise is one of the main factors of effectiveness 8 on this fact so that exercise with high intensity in all three exercise models of the present study increase adiponectin level and wasn't different in exercises. Besides, results of the present research showed that 8 weeks resistance exercise in exercise groups significantly reduced serum leptin which was in agreement with results of Wandell et al. (13). Other probable mechanisms to justify serum leptin level reduction in resistance exercises can reduce body fats and its storages by this exercises. Consequently, resistance exercises can motivate muscular protein synthesis so increase muscles of body. This fact energy consumption of resting time, 30 so body fat mass reduces and leptin secretion in people decreases $(14,15)$. In this regard, Ryan et al. showed that adiponectin plasma levels have negative correlation with fat $\%$ and leptin (12). One of the probable mechanisms to reduce leptin in resistance exercises is changes in testosterone. So that was indicated exercise and body activity increase LH secretion and consequently testosterone secretion in body by motivating HPG vector and increase Gonadotropin Releasing Hormone (GnRH) (16). Testosterone as a controller factor of building leptin in adipose tissue reduces leptin level (17). Therefore, increase in serum testosterone level 
can be attributed to reduction of leptin level in responding to exercises in present study. Simpson and Singh (2008) have claimed that the type of aerobic exercise and resistance training programs that are sufficiently intense performed in appropriate duration and result in significant decrease of body fat percent may result in significant increase of adiponectin level and Decrease in level of leptin in blood circulation (18). In addition, resistance training programs increase glucose removal by peripheral tissues in response to acidosis, lactate accumulation, increase of sympathy adrenal, cost of energy, glycogen depletion and by control of glycolysis increases insulin sensitivity, thus the improvement of body weight (increase of muscle mass and fat tissue decrease) results in plasma increase of adiponectin and Decreasing in levels of leptin (19). Therefore, the increase in the muscle mass in the combination training and decrease of body fat percent in the lower and upper extremities training groups observed in this research may have contributed to the increase of adiponectin and Decrease in level of leptin in these groups. Therefore, increase in serum adiponectin level and Decrease in level of leptin can be attributed to change in body composition in responding to exercises in present study.

\section{CONCLUSION}

The findings of the present study showed that levels of visfatin, glucose, body mass index and body fat percentage in the group of concurrent training were lower than that in groups of resistance and endurance training, and also insulin resistance of concurrent training were lower in than only endurance training group, showing that the effect of semi-insulin is more in concurrent training group. According to the results, probably, concurrent training had more effect on body composition, glucose metabolism and insulin resistance adjustment which can be effective in preventing obesity and adipokines' adjustment.

\section{APPLICABLE REMARKS}

- Resistance Training could be an appropriate method for increase in adiponectin and enhancement of body composition in young Men.

- Resistance Training could be an appropriate for increase of 1RM in Young Men.

- Resistance Training could be a reliable effective method in decreasing leptin.

\section{ACKNOWLEDGEMENT}

This research article is a part of doctor of philosophy $(\mathrm{PhD})$ dissertation in exercise physiology at Tehran Islamic Azad University that was made possible by the personal expenses paid by the researcher. No part of the project was sponsored by any corporation of agency. The researcher would like to take the opportunity to express his appreciation to his research advisor and counselors in addition to the generous contribution of the participants. The researcher also wishes to thank the personnels who performed the lab analysis in Kavesh Laboratory of Gorgan city.

\section{REFERENCES}

1. Calderon KS,Yucha CB, Schaffer SD. Obesity-Related Cardiovascular Risk Factors: Intervention Recommendations to Decrease Adolescent Obesity. J Pediatr Nurs. 2005;(20): 2-13. PMID: 15834354

2. DeMelo CM,Tirapegui J, Cohen D, Marchini JS, Ribeiro SM. Nutritional status and energy expenditure after a programme of nutrition education and combined aerobic/resistance training in obese women. Eur J Clin Nutr Metab.2010; 53(2): 1-7.

3. Shimada K, Miyazaki T, Daida H. Adiponectin and atherosclerotic disease. Clin Chim Acta. 2004; 344(1-2):1-12. PMID: 15149866

4. Fang X, Sweeney G. (2006). Mechanisms regulating energy metabolism by Adiponectin in obesity and diabetes. Biochem Soc Trans; 34(5): 708-801. PMID: 17052201 
5. Fatouros IG, Tournis S, Leontsini D, Jamurtas AZ, Sxina M, Thomakos P, Manousaki M, Douroudos I, Taxildaris $\mathrm{K}$, Mitrakou A. Leptin and adiponectin responses in overweight Inactive elderly following resistance training and detraining are Intensity related. J Clin Endocrinol Metab. 2005; 90(11):5970-7. PMID: 16091494

6. Ahmadizad S, AH Haghighi, Hamedinia MR. Effects of resistance versus endurance training on serum adiponectin and insulin resistance index. Eur J nol. 2007;157(5): 625-631. PMID: 17984242

7. taher Z, Hamedinia M, Haghighi A. Investigation of Effect of one Session Moderate and Heavy Resistance Exercise on Acute and Delayed Responses of Leptin, Insulin, Cortisol, Testosterone and 24- Hour Energy Expenditure in Healthy Men . Iranian Journal of Endocrinology and Metabolism. 2011; 13 (1) :67-73

8. Lau PWC, Kong Z, Choi CR, Yu CCW, Chan DFY, Sung RYT Leung Beeto W.C. . Effects of short-term resistance training on serum leptin levels obese adolescents. J Exer Sci Fitness. 2010; 8(1):54-60.

9. Ibáñez $\mathbf{J}^{1}$, Izquierdo M, Martínez-Labari C, Ortega F, Grijalba A, Forga L, Idoate F, García-Unciti M, FernándezReal JM, Gorostiaga EM. Resistance Training Improves Cardiovascular Risk Factors in Obese Women Despite a Significative Decrease in Serum Adiponectin Levels. Obesity (Silver Spring). 2010;18(3):535-41. PMID: 19713947

10. Calder AW, Chilibeck PD, Webber CE, Sale DG. Comparison of Whole and Split Weight Training Routines in Young Women, Can J Appl Physiol. 1994;19(2):185-99. PMID: 8081322

11- Balducci $S^{1}$, Zanuso S, Nicolucci A, Fernando F, Cavallo S, Cardelli P, Fallucca S, Alessi E, Letizia C, Jimenez A, Fallucca F, Pugliese G. Anti-inflammatory effect of exercise training in subjects with type 2 diabetes and the metabolic syndrome is dependent on exercise modalities and independent of weight loss. Nutr Metab Cardiovasc Dis. 2010;20(8):608-17. PMID: 19695853

12. Klimcakova $E^{1}$, Polak J, Moro C, Hejnova J, Majercik M, Viguerie N, Berlan M, Langin D, Stich V. Dynamic strength training improves Insulin sensitivity without altering plasma levels and gene Expression of adipokines in subcutaneous adipose tissue in obese men. J Clin Endocrinol Metab. J Clin Endocrinol Metab. 2006;91(12):510712. PMID: 16968804

13. Wandell P E, Arnlov J, Andreasson A N, Andersson K, Tornkvist L, Carlsson A C. Effects of tactile massage on metabolic biomarkers in patientswith type 2 diabetes. Diabetes and Metabolism. 2013; 39(5): 411-7.

14. Ara I, Prerez-Gomez J, Vicente-Rodriguez G, Chavarren J, Dorado C, Calbet JÁ. Serum free testosterone, leptin and soluble leptin receptor changes in a 6-week strength-training programme. Br J Nutr. 2006;96(6):1053-9. PMID: 17181880

15. Ronti T, Lupattelli G, Mannarino E. The endocrine function of adipose tissue: an update. Clin Endocrinol (Oxf). 2006;64(4):355-65. PMID: 16584505

16-Brand JS, van der Tweel I, Grobbee DE, Emmelot-Vonk MH, van der Schouw YT.Testosterone, sex hormonebinding globulin and the metabolic syndrome: a systematic review and meta-analysis of observational studies Int J Epidemiol. 2011;40(1):189-207. PMID: 20870782

17- Ahtiainen JP, Pakarinen A, Alen M, Kraemer WJ, Häkkinen K. Muscle hypertrophy, hormonal adaptations and strength development during strength training in strength-trained and untrained men. Eur J Appl Physiol. 2003;89(6):555-63. PMID: 12734759

18. Simpson KA, Singh MA. Effects of exercise on adiponectin; a systematic review. Obesity (Silver Spring) 2008; 16: 241-56.

19- Cnop M, Havel PJ, Utzschneider KM, Carr DB, Sinha MK, Boyko EJ, Retzlaff BM, Knopp RH, Brunzell JD, Kahn SE. . Relationship of adiponectin to body fat distribution, insulin sensitivity and plasma lipoproteins: evidence for independent roles of age and sex. Diabetologia. 2003;46(4):459-69. PMID: 12687327 